\title{
Pleomorphic Adenoma of Parotid in a Young Patient
}

Sangeeta R. Patankar, Megha A. Meshram, Vasant Shewale', Gokul S.

From the Department of Oral Pathology \& Microbiology and Department of Oral \& Maxillofacial Surgery'; Y.M.T. Dental College \& Hospital, Navi Mumbai, India.

\section{Abstract:}

Salivary gland tumors are rare, comprising less than $3 \%$ of all neoplasia of head and neck region. Pleomorphic adenoma, is the most common salivary gland tumor, accounting for about $60 \%$ to $80 \%$ of the benign tumors of the salivary glands and for $60 \%$ to $70 \%$ of all the parotid tumors. Pleomorphic adenoma exhibits wide cytomorphologic and architectural diversity. It is found more commonly between fifth and sixth decades of life, female to male ratio being 2:1. Early diagnosis of this benign neoplasm is important as it has a high rate of recurrence and chances of malignant transformation. Due to few symptoms and the possibility of extension into a hidden site, such as the parapharyngeal space, the tumor can grow for a long time before being diagnosed, and the potential risk of malignant transformation increases over the years with an incidence of $1-7 \%$. We present a case of pleomorphic adenoma diagnosed early in a young patient.

Key words: Pleomophic, Adenoma, Salivary Glands, Parotid Neoplasms, Neoplasm Recurrence.

\section{Introduction}

Salivary gland tumors are rare and make up about $3 \%$ of all neoplasms of the head and neck [1]. The parotid, submandibular glands, and the minor salivary glands of the palate are commonly involved, and the sublingual gland is rarely affected $[1,2]$. Pleomorphic adenoma occurs most commonly in the major salivary glands, $63 \%$ arising in the parotid gland [2], and most of these are seen in the superficial lobe [3]. Right side involvement is more common than left side and female to male ratio is $2: 1$. Its occurrence is rare in children and young adults and is found more commonly between fourth to sixth decades of life [2]. While pleomorphic adenomas are benign, they have a propensity for recurrence. Additionally, they have the risk of malignant transformation over time. These characteristics of pleomorphic adenoma necessitate their accurate diagnosis and appropriate management. We present one such case of pleomorphic adenoma diagnosed early in a young patient.

\section{Case Report}

A 24 year old male patient presented with a well-defined swelling just below left earlobe near the angle of mandible for last 3 years. The swelling was asymptomatic and medical history was noncontributory. On careful

Corresponding Author: Dr. Megha A Meshram

Email: megs22635@yahoo.com

Received: March 22, 2013 | Accepted: April 25, 2013 | Published Online: May 20, 2013

This is an Open Access article distributed under the terms of the Creative Commons Attribution License (creativecommons.org/licenses/by/3.0)

Conflict of interest: None declared | Source of funding: Nil | DOl: http://dx.doi.org/10.17659/01.2013.0035 
clinical examination, swelling of $3.5 \times 2 \mathrm{~cm}$ size was noticed below the earlobe near the angle of the mandible [Fig.1]. It was well circumscribed, superficial, firm in consistency, mobile and was not attached to the skin. Overlying skin was normal in color and texture. Left submandibular lymph nodes were palpable, nontender and mobile. Orthopantomograph showed no significant findings. A clinical diagnosis of chronic localized lymphadenopathy was considered along with differential diagnosis of Pleomorphic adenoma and Warthin's tumor in view of its location. The lesion was surgically excised and a mass of about $1.4 \times 2.2 \mathrm{~cm}$ was obtained.

On gross examination, it was ovoid, lobulated well defined and encapsulated mass. Areas of hemorrhages and fatty tissue were present on the surface. Histopathological examination showed an encapsulated mass lined by incomplete fibrous capsule with adjacent normal salivary gland tissue. The epithelial cells were arranged in nests and cords in the myxochondroid stroma. The myxoid and chondroid areas formed the predominant part of the tumor [Fig.2]. Higher magnification showed the tumor cells arranged in various forms such as tubules,

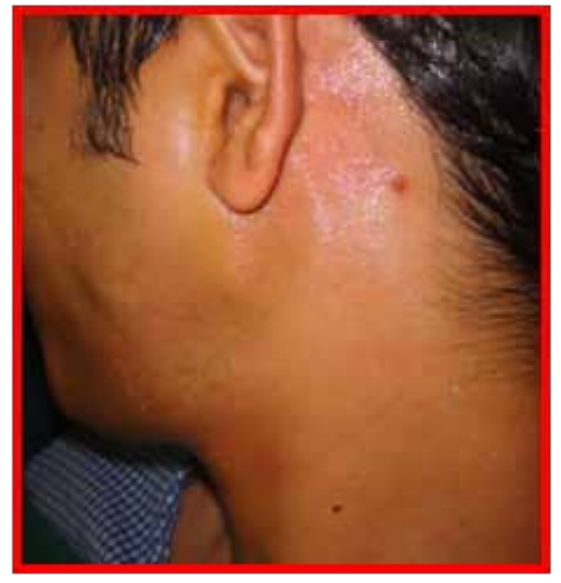

Fig.1: Slight elevation observed below the earlobe.
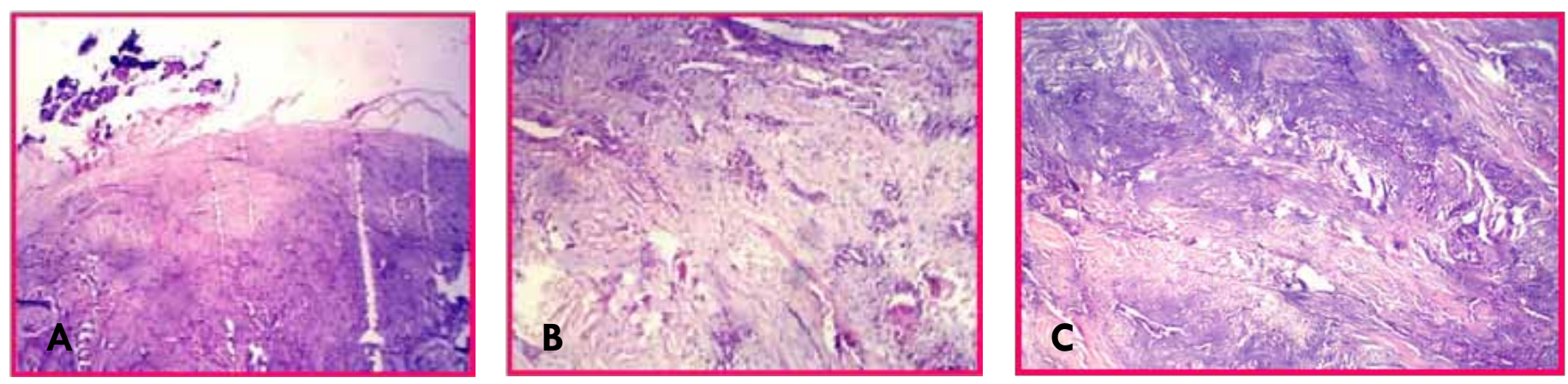

Fig.2: $H$ \& $E$ stained section in lower magnification showing A) tissue lined by incomplete fibrous capsule with adjacent normal serous glands; B) epithelial cells are arranged in nests and cords in myxo-chondroid stroma; C) myxoid and chondroid areas form the predominant part. 
ducts and nests [Fig.3]. Two types of cells were present in the tumor; cuboidal cells with eosinophillic cytoplasm and numerous myoepithelial cells with scanty cytoplasm. Cystic spaces with eosinophillic coagulum were also present [Fig.4]. With all these classic histopathological features, final diagnosis of pleomorphic adenoma was confirmed.

\section{Discussion}

Pleomorphic adenoma, also known as benign mixed tumor, is the most common salivary gland neoplasm and accounts for $60 \%$ of all benign salivary gland tumors [1]. It most commonly presents in middle age and is common in women [3]. Pleomorphic adenoma occurs most commonly in the major salivary glands, $63 \%$ arising in the parotid gland [1], and most of these arise in the superficial lobe [3]. It appears as a firm, painless and slow growing mass that is commonly diagnosed long after the lesion has started [4].

Subhashraj K. has performed a 15 year study on 684 cases of salivary gland tumors to analyze the incidence

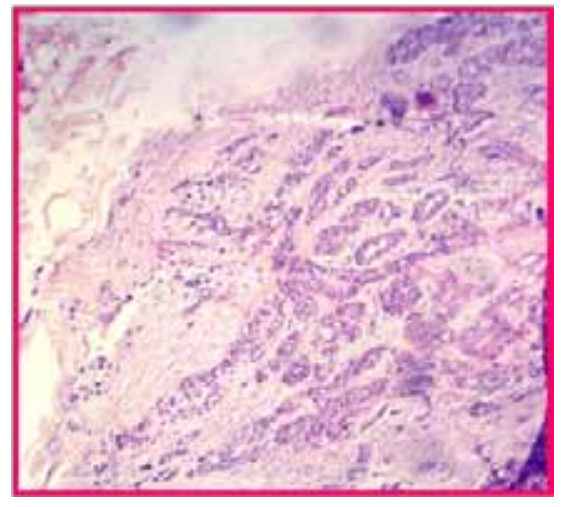

Fig.3: $H$ \& $E$ stained section in higher magnification showing tumor cells arranged in various forms such as tubules, ducts and nests in a surrounding myxoid stroma.

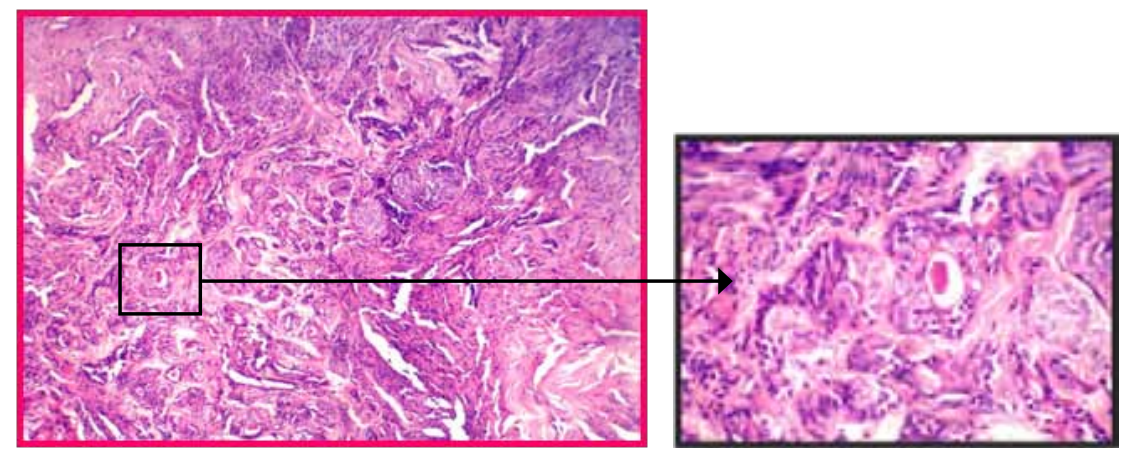

Fig.4: $H$ \& E stained section in higher magnification showing few cuboidal cells with eosinophilic cytoplasm and numerous myoepithelial cells with scanty cytoplasm; an area of cystic degeneration with eosinophillic coagulum is also seen. 
and distribution in India. He reported that Pleomorphic adenoma is the most common pathology of the major salivary gland tumor, accounting for $86 \%$ of all benign tumours [1]. All epidemiological studies on the salivary gland tumors have shown a pronounced predominance of pleomorphic adenoma [1,5]. Although the etiology of pleomorphic adenoma is unknown, the incidence of this tumor has been found to increase 15-20 years after exposure to radiation. One study suggests that the simian virus (SV40) may play a causative role in the development of pleomorphic adenoma [6].

Pleomorphic adenoma presents clinically as a solitary, painless, slow-growing, well-circumscribed parotid or preauricular lump. Its size at presentation varies considerably and depends on the duration of lesion [3]. Common age of presentation is 5 th \& 6th decade and has slight female predilection in contrast to the present case where the patient was male and quite younger, in his early 3rd decade of life.

It is usually encapsulated when it arises in the major salivary glands, but not in the minor salivary glands. Cells of epithelial origin give rise to ductal structures and are closely intermingled with mesenchymal elements that may give rise to myxoid, hyaline, cartilaginous, and osseous change [7]. The epithelial component consists of epithelial and myoepithelial cells with divergent growth patterns, including trabecular, tubular, solid, cystic, and papillary architecture [8].

Pure epithelial cells are mainly cuboidal. Cells that exhibit myoepithelial features may have plasmacytoid, epithelioid, spindle, oncocytic, or clear cell morphology $[8,9]$. Some authors have found that a prominent hyalinized stroma is correlated with tumors that may have a propensity for malignant change. Necrosis and increased mitosis are not commonly encountered in pleomorphic adenoma; when they do occur, a thorough search for evidence of malignant change is necessary [10].

Immunohistochemistry (IHC) may be supportive and helpful in delineating the different cell types and components, as well as in differentiating pleomorphic adenoma from other tumors. The markers which can be used are Keratin, Cam 5.2 and EMA, P-63, Calponin, Maspin, S-100, HHF-35, Muscle-specific actin, Glial fibrillary acidic protein, BMP and Aggrecan [1 1]. Most cases of pleomorphic adenomas (70\%) show cytogenetic aberrations. PLAG1 and HMGA2 gene translocations have been identified as tumor-specific in pleomorphic adenoma [12]. The treatment of pleomorphic adenoma includes surgical excision which could be by partial or total parotidectomy [13]. Since these tumors are radio-resistant, use of radiotherapy is of little benefit and is therefore contraindicated.

Local recurrence after excision has been reported in 1-5\% of cases [3]. Tumors with myxoid stroma demonstrate the highest incidence of capsular thinning or areas of capsular absence and pseudopodia formation [1 4] leading to increased propensity for recurrence. The present case also shows abundance of myxoid and chondroid stroma with thinning and irregularities in the capsule.

It is seen that recurrences are more common in younger patients than in older ones. McGregor AD et al studied 31 recurrent pleomorphic adenoma cases over 10 year period 1974-1983. They observed that these patients presented with their primary tumor at a significantly younger age than those who remained disease-free. Sixteen of 31 who subsequently developed recurrence were under 30 years of age at initial presentation. Of all patients under 30 years of age at first presentation, $42 \%$ subsequently developed recurrent disease [15]. 
This suggests that younger patients have higher risk of recurrence and hence should be followed up for a longer period. Since the patient in the present case is also young, long term follow up is required.

L. O. Redaelli de Zinis and co-workers in their study in 2005 observed that presence of a multinodular lesion and the type of intervention performed is seen to be significantly associated with a higher probability of recurrence [16]. The mucin 1 gene ( $M U C 1$ ) has been found to be related to the recurrence of pleomorphic adenoma and to be associated with malignant transformation of this tumor, with carcinoma cells over expressing MUC1 [17].

Malignant degeneration is possible within pleomorphic adenomas, and the incidence increases with tumor duration and size [18]. Histologic features suggestive of malignant transformation include extensive hyalinization, cellular atypism, necrosis, calcification, and invasion [14]. Longevity and recurrence are risk factors for malignant transformation, which occurs in $3-4 \%$ of cases [3]. The frequency of malignant tumours increased with age after the third decade and is maximal in the eighth decade [2]. These features highlight the importance of early diagnosis and management of pleomorphic adenoma. Since the present case was diagnosed early, it would probably reduce the chance of recurrence and malignant transformation.

\section{Conclusion}

The present case was diagnosed early in a young patient who came for a routine dental checkup. The mass was excised and confirmed as pleomorphic adenoma on histopathologic examination. Early diagnosis and timely management saved the patient of a disfigurement which is generally seen with this neoplasm. Patient showed uneventful healing and no sign of recurrence after eight months follow up. Since the patient was young, long term follow up is required.

\section{References}

1. Subhashraj K. Salivary gland tumors: a single institution experience in India. Br J Oral Maxillofac Surg. 2008;46:635-638.

2. Eveson JW, Cawson RA. Salivary gland tumours. A review of 2410 cases with particular reference to histological types, site, age and sex distribution. J Pathol.1985;146:51-58.

3. Lingam RK, Daghir AA, Nigar E, Abbas SAB, Kumar M. Pleomorphic adenoma (benign mixed tumour) of the salivary glands: its diverse clinical, radiological, and histopathological presentation. $\mathrm{Br} J$ Oral and Max Surg. $2011 ; 49: 14-20$.

4. Terán E C, Villasana EM. Lip pleomorphic adenoma. Rev Odonto Mex. 2012;16:102-104.

5. Spiro RH. Salivary neoplasms: overview of a 35-year experience with 2,807 patients. Head Neck Surg. 1986;8:177-184.

6. Martinelli $M$, Martini $F$, Rinaldi $E$, et al. Simian virus 40 sequences and expression of the viral large $T$ antigen oncoprotein in human pleomorphic adenomas of parotid glands. Am J Pathol. 2002;161:11271133.

7. Ito FA, Jorge J, Vargas PA, Lopes MA. Histopathological findings of pleomorphic adenomas of the salivary glands. Med Oral Patol Oral Cir Bucal. 2009;1 4:E57-E61. 
8. Batsakis JG, Kraemer B, Sciubba JJ. The pathology of head and neck tumors: the myoepithelial cell and its participation in salivary gland neoplasia, Part 17. Head Neck Surg. 1983;5:222-233.

9. Dardick I, van Nostrand AW, Phillips MJ. Histogenesis of salivary gland pleomorphic adenoma (mixed tumor) with an evaluation of the role of the myoepithelial cell. Hum Pathol. 1982;13:62-75.

10. Auclair PL, Ellis GL. Atypical features in salivary gland mixed tumors: their relationship to malignant transformation. Mod Pathol. 1996;9:652-657.

11. Nishimura T, Furukawa M, Kawahara E, Miwa A. Differential diagnosis of pleomorphic adenoma by immunohistochemical means. J Laryngol Otol. 1991;105:1057-1060.

12. Matsuyama A, Hisaoka M, Nagao Y, Hashimoto H. Aberrant PLAG1 expression in pleomorphic adenomas of the salivary gland: a molecular genetic and immunohistochemical study. Virchows Arch. 2011 ;458:583592.

13. Bradley PJ. Pleomorphic salivary adenoma of the parotid gland: which operation to perform? Curr Opin Otolaryngol Head Neck Surg. 2004;1 2:69-70.

14. Stennert E, Guntinas-Lichius O, Klussmann JP, et al. Histopathology of pleomorphic adenoma in the parotid gland: a prospective unselected series of 100 cases. Laryngoscope. 2001;111:2195-2200.

15. McGregor AD, Burgoyne M, Tan KC. Recurrent pleomorphic salivary adenoma--the relevance of age at first presentation. Br J Plast Surg. 1988;41:177-181.

16. L. O. Redaelli de Zinis, M Piccioni, AR Antonelli, P Nicolai. Management and prognostic factors of recurrent pleomorphic adenoma of the parotid gland: personal experience and review of the literature. Eur Arch Otorhinolaryngol. 2008;265:447-452.

17. Soares $A B$, Demasi AP, Altemani $A$, de Araújo VC. Increased mucin 1 expression in recurrence and malignant transformation of salivary gland pleomorphic adenoma. Histopathology. 201 1;58:377-382.

18. Spiro RH, Huvos AG, Strong EW. Malignant mixed tumor of salivary origin: a clinicopathologic study of 146 cases. Cancer. 1977;39:388-396. 